\title{
Association of CCL2, CCR2 and CCL5 genetic polymorphisms with the development and progression of benign prostatic hyperplasia
}

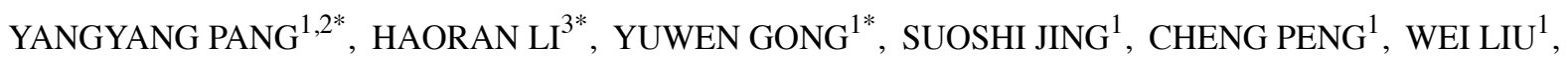 \\ YOULI ZHAO $^{1}$, HANZHANG WANG ${ }^{4}$, DHARAM KAUSHIK ${ }^{4}$, \\ RONALD RODRIGUEZ ${ }^{4}$ and ZHIPING WANG ${ }^{1}$
}

\begin{abstract}
${ }^{1}$ Institute of Urology, Lanzhou University Second Hospital, Key Laboratory of Urological Diseases in Gansu Province, Gansu Nephro-Urological Clinical Center, Lanzhou, Gansu 730030; ${ }^{2}$ Department of Urology, Jiading District Central Hospital Affiliated Shanghai University of Medicine and Health Sciences, Shanghai 201800; ${ }^{3}$ Department of Gynecologic Oncology,

Fudan University Shanghai Cancer Center, Department of Oncology, Shanghai Medical College,

Fudan University, Shanghai 200032, P.R. China; ${ }^{4}$ Department of Urology, University of Texas

Health Science Center San Antonio, San Antonio, TX 78229, USA
\end{abstract}

Received August 12, 2018; Accepted January 25, 2019

DOI: $10.3892 /$ or.2019.7002

\begin{abstract}
Benign prostatic hyperplasia (BPH) is a common chronic disease in older males. The pathogenesis of BPH remains elusive but may be associated with chronic inflammation. Chemokines and chemokine receptors have been implicated as critical mediators in the immune response and inflammatory processes. In the present study, the aim was to evaluate the association of three polymorphisms in chemokine genes, namely C-C motif chemokine ligand (CCL) 2 rs1024611, CC chemokine receptor 2 (CCR2) rs1799864 and CCL5 rs2107538, with BPH risk. These polymorphisms were genotyped in 109 patients with BPH and 160 control subjects, using the polymerase chain reaction and multiple ligase detection reaction method. The CCL5 rs2107538 polymorphism was identified to be associated with a significantly lower risk of BPH [A/G vs. G/G: Odds ratio
\end{abstract}

Correspondence to: Professor Zhiping Wang, Institute of Urology, Lanzhou University Second Hospital, 82 Cuiying Gate, Lanzhou, Gansu 730030, P.R. China

E-mail: erywzp@1zu.edu.cn

*Contributed equally

Abbreviations: $\mathrm{BPH}$, benign prostatic hyperplasia; CCL2, C-C motif chemokine ligand 2; CCR2, C-C motif chemokine receptor 2; CCL5, C-C motif chemokine ligand 5; SNP, single nucleotide polymorphism; OABSS, Overactive Bladder Symptom Score; IPSS, International Prostate Symptom Score; PSA, prostate specific antigen; QOL, quality of life; Qmax, peak urinary flow rate; $\mathrm{VV}$, voided volume; PV, prostate volume; PVR, post-void residual volume

Key words: benign prostatic hyperplasia, CCL2, CCR2, CCL5, polymorphism
$(\mathrm{OR})=0.37,95 \%$ confidence interval $(\mathrm{CI})=0.17-0.78 ; \mathrm{A} / \mathrm{A}+\mathrm{A} / \mathrm{G}$ vs. $\mathrm{G} / \mathrm{G}$ : $\mathrm{OR}=0.39,95 \% \mathrm{CI}=0.19-0.79$; $\mathrm{A}$ vs. $\mathrm{G}: \mathrm{OR}=0.58$, $95 \% \mathrm{CI}=0.35-0.96)$. However, this polymorphism was also associated with the development of larger prostate volumes in patients with $\mathrm{BPH}(\mathrm{A} / \mathrm{G}$ vs. $\mathrm{G} / \mathrm{G}$ : $\mathrm{OR}=3.02,95 \% \mathrm{CI}=1.28-7.11$; $\mathrm{AA}+\mathrm{AG}$ vs. $\mathrm{GG}$ : $\mathrm{OR}=2.83,95 \% \mathrm{CI}=1.28-6.26$; $\mathrm{A}$ vs. $\mathrm{G}$ : $\mathrm{OR}=1.94,95 \% \mathrm{CI}=1.08-3.49)$. The CCR2 rs1799864 polymorphism was associated with lower International Prostate Symptom Score values $(\mathrm{A} / \mathrm{A}+\mathrm{A} / \mathrm{G}$ vs. $\mathrm{G} / \mathrm{G}$ : $\mathrm{OR}=0.39,95 \% \mathrm{CI}=0.17-0.91$; A vs. G: $\mathrm{OR}=0.43,95 \% \mathrm{CI}=0.20-0.90)$ and low $\mathrm{Qmax}(\mathrm{A} / \mathrm{G}$ vs. $\mathrm{G} / \mathrm{G}: \mathrm{OR}=0.38,95 \% \mathrm{CI}=0.16-0.92 ; \mathrm{AA}+\mathrm{AG}$ vs. $\mathrm{GG}: \mathrm{OR}=0.39$, $95 \% \mathrm{CI}=0.17-0.91)$ in the patients. No association was observed between the CCL2 rs1024611 polymorphism and BPH. These results suggest that the CCR2 and CCL5 genes may contribute to the occurrence and progression of $\mathrm{BPH}$.

\section{Introduction}

Benign prostatic hyperplasia (BPH) is a condition comprising a series of lower urinary tract symptoms caused by the non-malignant enlargement of the prostate gland, which is a common condition in older men (1). The proportion of men older than 50 years with BPH increases with age, with an incidence of $>50 \%$ in men aged 60-69 years and 90\% in those aged 70-89 years (2). However, the etiology of BPH remains unclear. Certain studies have suggested a possible role of chronic inflammation in the pathogenesis and progression of BPH (3). A number of studies have sought the association between chronic inflammation and $\mathrm{BPH}$, speculating that $\mathrm{BPH}$ is an immune-mediated inflammatory disease (4-6). Prostate inflammation involves a complex network of cytokines and inflammatory mediators. This network leads to the sustained mitogenic stimulation of epithelial and stromal cells, eventually resulting in BPH (6).

Chemokines are chemotactic cytokines produced in activated innate immune cells, and are potent inducers of 
leukocytes, including neutrophils and monocytes, acting as mediators in acute and chronic inflammation. Through interaction with their receptors, chemokines regulate the directional movement of various immune cells in tissues and organs so as to maintain the homeostasis of the local microenvironment (7). C-C motif chemokine ligand (CCL2), also known as monocyte chemoattractant protein 1 , is a member of the $\mathrm{CC}$ chemokine family and regulates the accumulation of monocytes, macrophages and other inflammatory cells at sites of inflammation by binding with $\mathrm{CC}$ chemokine receptor 2 (CCR2) (8). CCL5, also known as regulated upon activation, normally T-expressed, and presumably secreted, is another cytokine that belongs to the CC chemokine family. CCL5 is chemotactic for $\mathrm{T}$ cells, eosinophils and basophils, and serves an active role in the recruitment of leukocytes to inflammatory sites (9). Prostate epithelial and stromal cells are able to secrete high levels of CCL2 and CCL5 in response to inflammatory stimulation. By binding with their receptors, they can stimulate the growth of epithelial cells through paracrine or autocrine pathways (10). Increased concentrations of CCL2 and CCL5, and the increased production of proinflammatory cytokines, such as interleukin (IL)6 and IL8, in the prostate gland, may further lead to the accumulation of inflammatory cells, resulting in a positive feedback loop that induces BPH (10).

A number of studies have investigated the association between single nucleotide polymorphisms (SNPs) in chemokines and their receptors, and prostate cancer (11-14). However, few studies have assessed the association of chemokines and their receptors with BPH risk. Therefore, in the present study, three common functional polymorphisms (CCL2 rs1024611, CCR2 rs1799864 and CCL5 rs2107538) were selected and their associations with the development and progression of $\mathrm{BPH}$ were investigated.

\section{Materials and methods}

Study subjects. A total of 269 subjects were enrolled in the present study between January 2014 and December 2015. These subjects comprised 109 patients with $\mathrm{BPH}$ and 160 healthy males as controls. The patients were diagnosed with BPH by at least two attending physicians in the Department of Urology, Lanzhou University Second Hospital (Lanzhou, China) according to their clinical features. For all patients, lower urinary tract symptoms were quantified using the International Prostate Symptom Score (IPSS), IPSS-quality of life (QOL) score and Overactive Bladder Symptom Score (OABSS) $(15,16)$. The serum prostate-specific antigen (PSA) level was determined by ELISA in each subject. Patients with serum PSA levels $\geq 4 \mathrm{ng} / \mathrm{ml}$ were further tested to exclude prostate cancer by digital rectal examination and transrectal ultrasound-guided prostate biopsy. The peak urinary flow rate (Qmax) and the voided volumes were measured for all patients using an uroflowmetry system (ZNC 961A; Yongxin Medical Equipment Co., Ltd., Jiangsu, China). The prostate volumes (PVs) of the patients were calculated using transrectal ultrasonography and the post-void residual volumes were measured by transabdominal ultrasonography. Subjects with a history of urinary tract infection, prostate cancer, neurogenic bladder dysfunction, acute/chronic prostatitis, uncontrolled diabetes, lower tract surgery or cardiovascular disease were excluded from the study. Based on previous studies $(17,18)$, all the BPH cases were separated into two groups based on the measurements of each parameter: IPSS, $<20$ vs. $\geq 20$; PSA, $<1.5$ vs. $\geq 1.5 \mathrm{ng} / \mathrm{ml}$; PV, $<30$ vs. $\geq 30 \mathrm{ml}$; and Qmax, $<10$ vs. $\geq 10 \mathrm{ml} / \mathrm{sec}$.

The healthy controls recruited in the present study were older males visiting the physician for routine checkups. They all underwent screening, had normal serum PSA levels $(<4.0 \mathrm{ng} / \mathrm{ml})$, and exhibited no evidence of any of the aforementioned diseases.

All the participants provided written informed consent prior to participation. The present study was approved by the Ethics Committee at Lanzhou University Second Hospital.

SNP selection and genotyping. In the present study, three SNPs (CCL2 rs1024611, CCR2 rs1799864, and CCL5 rs2107538) that have previously been demonstrated to have an association with prostate cancer were selected (11-14). Peripheral venous blood samples from all participants were collected in EDTA blood collection tubes and stored at $-80^{\circ} \mathrm{C}$ prior to the extraction of genomic DNA. Genomic DNA was extracted from the blood using the Tiangen DNA extraction kit (Tiangen Biotech Co., Ltd., Beijing, China). The SNPs were genotyped using the polymerase chain reaction (PCR) and multiple ligase detection reaction (LDR) system at Shanghai Biowing Applied Biotechnology Co., Ltd. (Shanghai, China). Based on the sequence information of chemokine genes on the NCBI website (https://www.ncbi.nlm.nih.gov/), PCR primers and LDR probes (Table I) were designed using Primer Premier version 5.0 software (Premier Biosoft International, Palo Alto, CA, USA) and synthesized by Shanghai Biowing Applied Biotechnology Co., Ltd. (Shanghai, China). PCR conditions consisted of 40 cycles at $94^{\circ} \mathrm{C}$ for $30 \mathrm{sec}, 53^{\circ} \mathrm{C}$ for $90 \mathrm{sec}$ and $65^{\circ} \mathrm{C}$ for $30 \mathrm{sec}$, followed by 1 cycle at $65^{\circ} \mathrm{C}$ for $10 \mathrm{~min}$ to terminate the reaction. LDR conditions consisted of 40 cycles at $94^{\circ} \mathrm{C}$ for $15 \mathrm{sec}$ and $50^{\circ} \mathrm{C}$ for $25 \mathrm{sec}$. The reagent used in these methods, such as DNA polymerase and DNA ligase were supplied by Shanghai Biowing Applied Biotechnology Co., Ltd. All PCR products were analyzed for quality and concentration using 3.0\% agarose gel electrophoresis. Visualization was achieved using ethidium bromide.

Functional annotation of selected SNP. To explore the potential function of the loci identified from the study, the online tool RegulomeDB (http://www.regulomedb.org/) was used to predict the putative function of the identified SNPs (19). Furthermore, assessment of whether the loci of genes may influence their corresponding mRNA expression levels was conducted using the online GTEx database (http://www.gtexportal.org/home/).

Statistical analysis. The genotype distributions of the SNPs in the patient and control groups were analyzed with a Chi-square test to investigate the Hardy-Weinberg equilibrium (HWE). Logistic regression models were used to estimate the main effects of the SNPs on BPH risk, assuming an additive and dominant model of inheritance, and adjusting for age as a covariable. Logistic regression analysis was also performed on the combined results of the three polymorphisms. The risk 
Table I. Primer and probe sequences used in PCR-LDR.

\begin{tabular}{|c|c|c|c|}
\hline SNP & Primer & Primer sequence $\left(5^{\prime}-3^{\prime}\right)$ & PCR length (bp) \\
\hline CCL2 rs 1024611 & $\begin{array}{l}\text { Forward } \\
\text { Reverse }\end{array}$ & $\begin{array}{l}\text { cagtaaacacagggaaggtg } \\
\text { tcttgacagagcagaagtgg }\end{array}$ & 95 \\
\hline CCR2 rs 1799864 & $\begin{array}{l}\text { Forward } \\
\text { Reverse }\end{array}$ & $\begin{array}{l}\text { gctctactcgctggtgttc } \\
\text { agatcagagatggccaggt }\end{array}$ & 128 \\
\hline CCL5 rs2107538 & $\begin{array}{l}\text { Forward } \\
\text { Reverse }\end{array}$ & $\begin{array}{l}\text { caaggagtggcagttagga } \\
\text { tatccagaggaccetcctc }\end{array}$ & 170 \\
\hline
\end{tabular}

B, Probe sequences

\begin{tabular}{|c|c|c|c|}
\hline SNP & Probe & Probe sequence $\left(5^{\prime}-3^{\prime}\right)$ & LDR length (bp) \\
\hline \multirow[t]{3}{*}{ CCL2 rs 1024611} & Modify & $\begin{array}{l}\text { P-tcactttccagaagactttcttttcttttttttttttt } \\
\text { tttttttttttttttttttttttttttttttttttttttt-FAM }\end{array}$ & \\
\hline & $\mathrm{C}$ & $\begin{array}{l}\text { ttttttttttttttttttttttttttttttttttttttttttttttttttttttt } \\
\text { ttttttttttgagcagaagtgggaggcagacagctg }\end{array}$ & 170 \\
\hline & $\mathrm{T}$ & $\begin{array}{l}\text { tttttttttttttttttttttttttttttttttttttttttttttttttttttttt } \\
\text { tttttttttttgagcagaagtgggaggcagacagcta }\end{array}$ & 172 \\
\hline \multirow[t]{3}{*}{ CCR2 rs 1799864} & Modify & $\begin{array}{l}\text { P-gaccagcatgttgcccacaaaaccatttttttttttttt } \\
\text { tttttttttttttttttttttttttttttttttttttttt-FAM }\end{array}$ & \\
\hline & A & $\begin{array}{l}\text { tttttttttttttttttttttttttttttttttttttttttttttttttttttttt } \\
\text { tttttttttgcagtttattaagatgaggat }\end{array}$ & 165 \\
\hline & G & $\begin{array}{l}\text { tttttttttttttttttttttttttttttttttttttttttttttttttttttttt } \\
\text { tttttttttttgcagtttattaagatgaggac }\end{array}$ & 167 \\
\hline \multirow[t]{3}{*}{ CCL5 rs2107538 } & Modify & $\begin{array}{l}\text { P-taagatctgtaatgaataagcaggattttttttttttttt } \\
\text { ttttttttttttttttttttttttttttttttttttttt-FAM }\end{array}$ & \\
\hline & $\mathrm{C}$ & $\begin{array}{l}\text { tttttttttttttttttttttttttttttttttttttttttttttttttttttttt } \\
\text { ttttttttttttttttttccttccatggatgagggaaaggagg }\end{array}$ & 180 \\
\hline & $\mathrm{T}$ & $\begin{array}{l}\text { ttttttttttttttttttttttttttttttttttttttttttttttttttttttttttt } \\
\text { tttttttttttttttttttccttccatggatgagggaaaggaga }\end{array}$ & 182 \\
\hline
\end{tabular}

PCR, polymerase chain reaction; LDR, ligase detection reaction; SNP, single nucleotide polymorphism; CCL, C-C motif chemokine ligand; CCR, CC chemokine receptor; P, primer; FAM, 5-carboxyfluorescein.

of BPH was evaluated by calculating the odds ratio (OR) and corresponding $95 \%$ confidence interval (CI). Stratified analyses were executed according to IPSS, PSA, PV and Qmax. Data obtained from the GTEx database were calculated and reported as the mean \pm standard deviation. Comparisons between different genotype groups were evaluated by one-way analysis of variance followed by Tukey's multiple comparison tests. All analyses were conducted using IBM SPSS version 20.0 software (IBM Corp., Armonk, NY, USA). $\mathrm{P}<0.05$ was considered to indicate a statistically significant difference.

\section{Results}

Clinical characteristics of the study subjects. The clinical characteristics of the 109 cases and 160 controls are presented in Table II. The mean age of the patients $(65.62 \pm 8.20$ years) was higher compared with that of the controls (54.51 \pm 3.87 years), and the association tests were adjusted accordingly.

HWE test. The genotype distributions of the SNPs in the disease and control groups were evaluated, and the results revealed good conformity to the HWE for all the SNPs $(\mathrm{P}>0.05)$. Therefore, the study population included in the present study is a typical Mendelian population (Table III).

Genetic association with BPH risk. The genotype distributions of the three studied genetic polymorphisms in the $\mathrm{BPH}$ and control groups are presented in Fig. 1. The specific associations between the SNPs and BPH risk were calculated (Table IV). The G/G, G/A and A/A genotype frequencies 


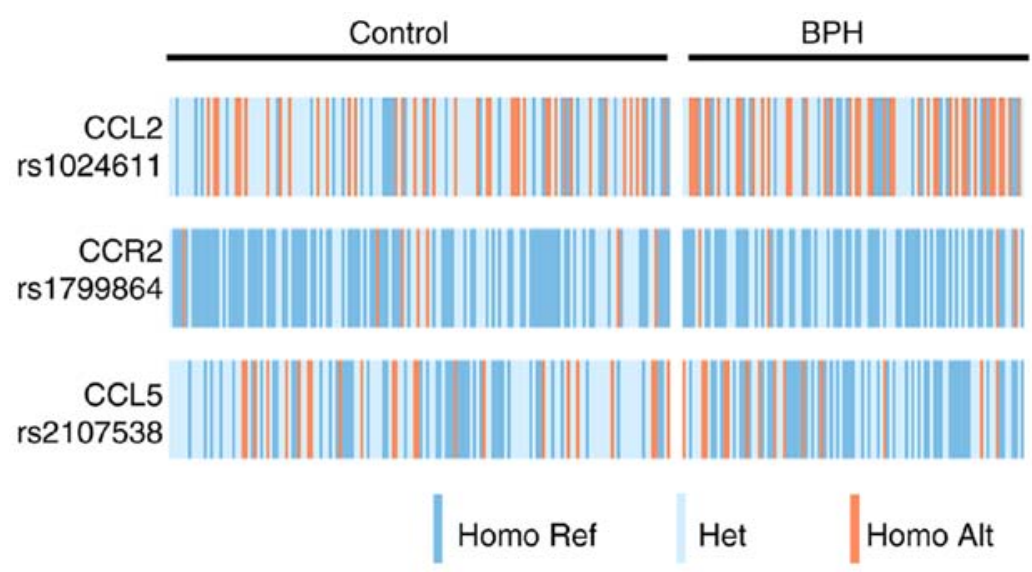

Figure 1. Genotype distributions of three single nucleotide polymorphisms in patients with BPH and healthy controls. BPH, benign prostatic hyperplasia; CCL, C-C motif chemokine ligand; CCR, CC chemokine receptor; Homo Ref, homozygous reference; Het, heterozygous; Homo Alt, homozygous alteration.

Table II. Clinical characteristics of all subjects.

\begin{tabular}{lcc}
\hline Characteristics & Cases & Controls \\
\hline No. of subjects & 109 & 160 \\
Age (years) & $65.62 \pm 8.20$ & $54.51 \pm 3.87$ \\
IPSS & $16.09 \pm 8.15$ & N/A \\
$0-19$ & $70(64.2)$ & N/A \\
$\geq 20$ & $39(35.8)$ & N/A \\
PSA (ng/ml) & $2.46 \pm 2.82$ & N/A \\
$<1.5$ & $52(47.7)$ & N/A \\
$\geq 1.5$ & $57(52.3)$ & N/A \\
PV $(\mathrm{ml})$ & $33.13 \pm 19.72$ & N/A \\
$<30$ & $56(51.4)$ & N/A \\
$\geq 30$ & $53(48.6)$ & N/A \\
Qmax (ml/sec) & $10.32 \pm 3.41$ & N/A \\
$<10$ & $39(35.8)$ & N/A \\
$\geq 10$ & $70(64.2)$ & N/A \\
OABSS & $4.96 \pm 3.19$ & N/A \\
QoL & $4.44 \pm 0.87$ & N/A \\
VV (ml) & $232.73 \pm 109.99$ & N/A \\
PVR (ml) & $27.57 \pm 43.10$ & N/A \\
\hline
\end{tabular}

IPSS, International Prostate Symptom Score; PSA, prostate-specific antigen; PV, prostate volume; Qmax, peak urinary flow rate; OABSS, Overactive Bladder Symptom Score; QoL, quality of life; VV, voided volume; PVR, post-void residual volume; N/A, not applicable. Values are presented as mean \pm standard deviation or as $\mathrm{n}(\%)$.

of the CCL5 rs2107538 polymorphism were $47.7,39.5$ and $12.8 \%$, respectively, in the patients, and $31.2,54.4$ and $14.4 \%$, respectively, in the controls. The allele frequencies of the patient and control groups were 67.4 and $58.4 \%$ for the $\mathrm{G}$ allele, and 32.6 and $41.6 \%$ for the A allele, respectively. The CCL5 rs2107538 polymorphism exhibited a significant association with a decreased risk of $\mathrm{BPH}$ in the heterozygous $(\mathrm{OR}=0.37$, 95\% $\mathrm{CI}=0.17-0.78)$, dominant $(\mathrm{OR}=0.39,95 \% \mathrm{CI}=0.19-0.79)$ and allelic form $(\mathrm{OR}=0.58,95 \% \mathrm{CI}=0.35-0.96)$. However, no significant differences in the genotype or allele distributions for the CCL2 rs1024611 and CCR2 rs1799864 polymorphisms were observed between the patients and controls.

Furthermore, all possible interactions of CCL5 rs2107538 with CCL2 rs1024611 and CCR2 rs1799864 gene polymorphisms were investigated. The combined genotype G/A-A/G of CCL5 rs2107538 and CCL2 rs1024611 exhibited a decreased risk of BPH. However, an increased risk of BPH was implied for the $\mathrm{G} / \mathrm{G}-\mathrm{G} / \mathrm{G}$ combination of these polymorphisms (Table V). The combined genotype G/A-G/G of CCL5 rs2107538 and CCR2 rs1799864 was also associated with a lower risk for BPH (Table VI). These results suggest that the interaction between the CCL5 rs2107538 and CCR2 rs1799864 polymorphisms may give rise to a synergistic effect.

Genetic association with clinicopathological characteristics. The associations between the three SNPs and the clinicopathological characteristics (IPSS, PSA, PV and Qmax) of the patients with BPH were investigated. The results indicated that CCR2 rs1799864 was significantly associated with IPSS, with a decreasing risk of high IPSS progression in the dominant $(\mathrm{OR}=0.39,95 \% \mathrm{CI}=0.17-0.91)$ and the allelic forms ( $\mathrm{OR}=0.43,95 \% \mathrm{CI}=0.20-0.90)$ (Table VII).

A significant association was detected between CCL5 rs2107538 and PV, as demonstrated in Table VIII, reflecting an increasing effect on the risk of large PV progression in the heterozygous $(\mathrm{OR}=3.02,95 \% \mathrm{CI}=1.28-7.11)$ and dominant model $(\mathrm{OR}=2.83,95 \% \mathrm{CI}=1.28-6.26)$. The allele frequency analysis confirmed that CCL5 rs2107538 was associated with PV (OR=1.94, 95\% CI=1.08-3.49). Furthermore, a significant association between CCR2 rs1799864 and Qmax ( $\geq 10$ vs. $<10 \mathrm{ml} / \mathrm{sec}$ ) was identified. Table IX demonstrates that CCR2 rs1799864 exhibited a decreasing risk of low Qmax progression in the heterozygous $(\mathrm{OR}=0.38,95 \% \mathrm{CI}=0.16-0.92)$ and dominant model $(\mathrm{OR}=0.39,95 \% \mathrm{CI}=0.17-0.91)$. However, no association was detected between the SNPs and PSA (Table X).

Functional prediction of genetic variants of CCL2, CCR 2 and CCL5. To investigate the role of the CCL2 rs1024611, CCR2 rs1799864 and CCL5 rs2107538 polymorphisms in the regulation of gene expression, a search of the GTEx database for data on mRNA expression was conducted (Fig. 2). For CCL5 
Table III. Exact Hardy-Weinberg equilibrium test for SNPs (n=269).

\begin{tabular}{|c|c|c|c|c|}
\hline SNP & Genotype or allele & All subjects & Cases & Controls \\
\hline \multirow[t]{5}{*}{ CCL2 rs 1024611} & $\mathrm{~A} / \mathrm{A}$ & 56 & 25 & 31 \\
\hline & $\mathrm{A} / \mathrm{G}$ & 135 & 44 & 91 \\
\hline & $\mathrm{G} / \mathrm{G}$ & 78 & 40 & 38 \\
\hline & A & 247 & 94 & 153 \\
\hline & G & 291 & 124 & 167 \\
\hline P-value & & 0.9 & 0.078 & 0.11 \\
\hline \multirow[t]{5}{*}{ CCR2 rs 1799864} & $\mathrm{G} / \mathrm{G}$ & 157 & 63 & 94 \\
\hline & $\mathrm{G} / \mathrm{A}$ & 101 & 42 & 59 \\
\hline & $\mathrm{A} / \mathrm{A}$ & 11 & 4 & 7 \\
\hline & G & 415 & 168 & 247 \\
\hline & A & 123 & 50 & 73 \\
\hline P-value & & 0.39 & 0.43 & 0.66 \\
\hline \multirow[t]{5}{*}{ CCL5 rs2107538 } & $\mathrm{G} / \mathrm{G}$ & 102 & 52 & 50 \\
\hline & $\mathrm{G} / \mathrm{A}$ & 130 & 43 & 87 \\
\hline & $\mathrm{A} / \mathrm{A}$ & 37 & 14 & 23 \\
\hline & $\mathrm{G}$ & 334 & 147 & 187 \\
\hline & A & 204 & 71 & 133 \\
\hline $\mathrm{P}$-value & & 0.7 & 0.28 & 0.15 \\
\hline
\end{tabular}

SNP, single nucleotide polymorphism; CCL, C-C motif chemokine ligand; CCR, CC chemokine receptor.

Table IV. Genotypes and allele frequencies of SNPs and their association with BPH risk.

\begin{tabular}{|c|c|c|c|c|c|c|}
\hline SNP & $\begin{array}{c}\text { Genotype or } \\
\text { allele }\end{array}$ & $\begin{array}{c}\text { Controls, } \\
\mathrm{n}=160(\%)\end{array}$ & $\begin{array}{c}\text { Cases } \\
\mathrm{n}=109(\%)\end{array}$ & Models & OR $(95 \% \mathrm{CI})^{\mathrm{a}}$ & P-value ${ }^{a}$ \\
\hline \multirow[t]{5}{*}{ CCL2 rs 1024611} & $\mathrm{~A} / \mathrm{A}$ & $31(19.4)$ & $25(22.9)$ & Heterozygous & $0.46(0.19-1.12)$ & 0.086 \\
\hline & $\mathrm{A} / \mathrm{G}$ & $91(56.9)$ & $44(40.4)$ & Homozygous & $1.27(0.49-3.28)$ & 0.62 \\
\hline & $\mathrm{G} / \mathrm{G}$ & $38(23.8)$ & $40(36.7)$ & Dominant & $0.68(0.30-1.56)$ & 0.37 \\
\hline & A & $153(47.8)$ & $94(43.1)$ & & 1 & \\
\hline & G & $167(52.2)$ & $124(56.9)$ & & $1.22(0.76-1.97)$ & 0.42 \\
\hline \multirow[t]{5}{*}{ CCR2 rs 1799864} & $\mathrm{G} / \mathrm{G}$ & $94(58.8)$ & $63(57.8)$ & Heterozygous & $1.11(0.54-2.27)$ & 0.78 \\
\hline & $\mathrm{G} / \mathrm{A}$ & $59(36.9)$ & $42(38.5)$ & Homozygous & $1.31(0.28-6.11)$ & 0.73 \\
\hline & $\mathrm{A} / \mathrm{A}$ & $7(4.4)$ & $4(3.7)$ & Dominant & $1.13(0.57-2.25)$ & 0.72 \\
\hline & $\mathrm{G}$ & $247(77.2)$ & $168(77.1)$ & & 1 & \\
\hline & A & $73(22.8)$ & $50(22.9)$ & & $1.12(0.64-1.98)$ & 0.69 \\
\hline \multirow[t]{5}{*}{ CCL5 rs2107538 } & $\mathrm{G} / \mathrm{G}$ & $50(31.2)$ & $52(47.7)$ & Heterozygous & $0.37(0.17-0.78)$ & 0.01 \\
\hline & $\mathrm{G} / \mathrm{A}$ & $87(54.4)$ & $43(39.5)$ & Homozygous & $0.47(0.16-1.35)$ & 0.16 \\
\hline & $\mathrm{A} / \mathrm{A}$ & $23(14.4)$ & $14(12.8)$ & Dominant & $0.39(0.19-0.79)$ & 0.008 \\
\hline & $\mathrm{G}$ & $187(58.4)$ & $147(67.4)$ & & 1 & \\
\hline & A & $133(41.6)$ & $71(32.6)$ & & $0.58(0.35-0.96)$ & 0.033 \\
\hline
\end{tabular}

SNP, single nucleotide polymorphism; BPH, benign prostatic hyperplasia; CCL, C-C motif chemokine ligand; CCR, CC chemokine receptor; OR, odds ratio; CI, confidence interval. ${ }^{\mathrm{a}} \mathrm{ORs}$ and $\mathrm{P}$-values were calculated by logistic regression with adjustment for age. Bold text indicates a statistically significant result.

rs2107538, the mRNA expression levels were downregulated following the $G>A$ conversion in whole blood cells $(P<0.001$,
Fig. 2C), but were not changed in prostate tissue $(\mathrm{P}=0.20$; data not shown). The CCL2 rs1024611 A>G and CCR2 rs1799864 
Table V. Association of BPH risk with different combinations of CCL5 rs2107538 and CCL2 rs1024611 genotypes.

\begin{tabular}{|c|c|c|c|c|}
\hline CCL5 rs2107538 and CCL2 rs1024611 & Controls, $\mathrm{n}=160(\%)$ & Cases, $\mathrm{n}=109(\%)$ & OR $(95 \% \mathrm{CI})^{\mathrm{a}}$ & P-value ${ }^{a}$ \\
\hline $\mathrm{G} / \mathrm{G}-\mathrm{A} / \mathrm{A}$ & $11(6.9)$ & $14(12.8)$ & 1 & \\
\hline $\mathrm{G} / \mathrm{G}-\mathrm{A} / \mathrm{G}$ & $37(23.1)$ & $16(14.7)$ & $0.26(0.07-1.01)$ & 0.05 \\
\hline $\mathrm{G} / \mathrm{G}-\mathrm{G} / \mathrm{G}$ & $2(1.2)$ & $22(20.2)$ & $10.83(1.50-78.13)$ & 0.018 \\
\hline $\mathrm{G} / \mathrm{A}-\mathrm{A} / \mathrm{A}$ & $12(7.5)$ & $7(6.4)$ & $0.65(0.12-3.53)$ & 0.62 \\
\hline $\mathrm{G} / \mathrm{A}-\mathrm{A} / \mathrm{G}$ & $44(27.5)$ & $20(18.3)$ & $0.20(0.05-0.77)$ & 0.019 \\
\hline $\mathrm{G} / \mathrm{A}-\mathrm{G} / \mathrm{G}$ & $31(19.4)$ & $16(14.7)$ & $0.28(0.07-1.11)$ & 0.07 \\
\hline $\mathrm{A} / \mathrm{A}-\mathrm{A} / \mathrm{A}$ & $8(5.0)$ & $4(3.7)$ & $0.13(0.02-1.10)$ & 0.06 \\
\hline $\mathrm{A} / \mathrm{A}-\mathrm{A} / \mathrm{G}$ & $10(6.2)$ & $8(7.3)$ & $0.55(0.11-2.83)$ & 0.47 \\
\hline $\mathrm{A} / \mathrm{A}-\mathrm{G} / \mathrm{G}$ & $5(3.1)$ & $2(1.8)$ & $0.46(0.04-5.00)$ & 0.52 \\
\hline
\end{tabular}

BPH, benign prostatic hyperplasia; CCL, C-C motif chemokine ligand; OR, odds ratio; CI, confidence interval. ${ }^{\mathrm{a} O R s}$ and P-values were calculated by logistic regression with adjustment for age. Bold text indicates a statistically significant result.

Table VI. Association of BPH risk with different combinations of CCL5 rs2107538 and CCR2 rs1799864 genotypes.

\begin{tabular}{|c|c|c|c|c|}
\hline CCL5 rs2107538 and CCR2 rs1799864 & Controls, $\mathrm{n}=160(\%)$ & Cases, $\mathrm{n}=109(\%)$ & OR $(95 \% \mathrm{CI})^{\mathrm{a}}$ & P-value ${ }^{a}$ \\
\hline $\mathrm{G} / \mathrm{G}-\mathrm{G} / \mathrm{G}$ & $30(18.8)$ & $30(27.5)$ & 1 & \\
\hline G/G-G/A & $18(11.2)$ & $20(18.3)$ & $0.77(0.26-2.33)$ & 0.65 \\
\hline $\mathrm{G} / \mathrm{G}-\mathrm{A} / \mathrm{A}$ & $2(1.2)$ & $2(1.8)$ & $1.51(0.11-20.36)$ & 0.76 \\
\hline $\mathrm{G} / \mathrm{A}-\mathrm{G} / \mathrm{G}$ & $53(33.1)$ & $26(23.9)$ & $0.29(0.11-0.78)$ & 0.014 \\
\hline $\mathrm{G} / \mathrm{A}-\mathrm{G} / \mathrm{A}$ & $31(19.4)$ & $16(14.7)$ & $0.45(0.15-1.37)$ & 0.16 \\
\hline $\mathrm{G} / \mathrm{A}-\mathrm{A} / \mathrm{A}$ & $3(1.9)$ & $1(0.9)$ & $0.26(0.02-3.76)$ & 0.32 \\
\hline $\mathrm{A} / \mathrm{A}-\mathrm{G} / \mathrm{G}$ & $11(6.9)$ & $7(6.4)$ & $0.36(0.08-1.56)$ & 0.17 \\
\hline $\mathrm{A} / \mathrm{A}-\mathrm{G} / \mathrm{A}$ & $10(6.2)$ & $6(5.5)$ & $0.42(0.08-2.28)$ & 0.32 \\
\hline $\mathrm{A} / \mathrm{A}-\mathrm{A} / \mathrm{A}$ & $2(1.2)$ & $1(0.9)$ & $0.94(0.07-12.16)$ & 0.96 \\
\hline
\end{tabular}

BPH, benign prostatic hyperplasia; CCL, C-C motif chemokine ligand; CCR, CC chemokine receptor; OR, odds ratio; CI, confidence interval . ${ }^{\mathrm{a}} \mathrm{ORs}$ and $\mathrm{P}$-values were calculated by logistic regression with adjustment for age. Bold text indicates a statistically significant result.

Table VII. Genotype and allele frequencies of SNPs and their association with IPSS.

\begin{tabular}{|c|c|c|c|c|c|c|}
\hline SNP & Genotype or allele & IPSS <20, n=70 (\%) & IPSS $\geq 20, n=39(\%)$ & Models & OR $(95 \% \mathrm{CI})^{\mathrm{a}}$ & $\mathrm{P}$-value ${ }^{\mathrm{a}}$ \\
\hline \multirow[t]{5}{*}{ CCL2 rs 1024611} & $\mathrm{~A} / \mathrm{A}$ & $14(20)$ & $11(28.2)$ & Heterozygous & $0.57(0.21-1.59)$ & 0.29 \\
\hline & $\mathrm{A} / \mathrm{G}$ & $30(42.9)$ & $14(35.9)$ & Homozygous & $0.69(0.25-1.92)$ & 0.47 \\
\hline & $\mathrm{G} / \mathrm{G}$ & $26(37.1)$ & $14(35.9)$ & Dominant & $0.63(0.25-1.56)$ & 0.32 \\
\hline & A & $58(41.4)$ & $36(46.2)$ & & 1 & \\
\hline & $\mathrm{G}$ & $82(58.6)$ & $42(53.8)$ & & $0.83(0.47-1.45)$ & 0.51 \\
\hline \multirow[t]{5}{*}{ CCR2 rs1799864 } & $\mathrm{G} / \mathrm{G}$ & $35(50)$ & $28(71.8)$ & Heterozygous & $0.44(0.19-1.03)$ & 0.06 \\
\hline & $\mathrm{G} / \mathrm{A}$ & $31(44.3)$ & $11(28.2)$ & Homozygous & $0.00(0.00-\mathrm{NA})$ & 1.00 \\
\hline & $\mathrm{A} / \mathrm{A}$ & $4(5.7)$ & $0(0)$ & Dominant & $0.39(0.17-0.91)$ & 0.026 \\
\hline & $\mathrm{G}$ & $101(72.1)$ & $67(85.9)$ & & 1 & \\
\hline & A & 39 (27.9) & $11(14.1)$ & & $0.43(0.20-0.90)$ & 0.024 \\
\hline \multirow[t]{5}{*}{ CCL5 rs 2107538} & $\mathrm{G} / \mathrm{G}$ & 37 (52.9) & $15(38.5)$ & Heterozygous & $1.73(0.73-4.08)$ & 0.21 \\
\hline & $\mathrm{G} / \mathrm{A}$ & $25(35.7)$ & $18(46.1)$ & Homozygous & $1.79(0.53-6.07)$ & 0.35 \\
\hline & $\mathrm{A} / \mathrm{A}$ & $8(11.4)$ & $6(15.4)$ & Dominant & $1.74(0.78-3.90)$ & 0.17 \\
\hline & $\mathrm{G}$ & 99 (70.7) & $48(61.5)$ & & 1 & \\
\hline & A & $41(29.3)$ & $30(38.5)$ & & $1.47(0.82-2.65)$ & 0.20 \\
\hline
\end{tabular}

SNP, single nucleotide polymorphism; IPSS, International Prostate Symptom Score; CCL, C-C motif chemokine ligand; CCR, CC chemokine receptor; OR, odds ratio; CI, confidence interval. ${ }^{a} \mathrm{ORs}$ and P-values were calculated by logistic regression with adjustment for age. Bold text indicates a statistically significant result. 
Table VIII. Genotype and allele frequencies of SNPs and their association with PV.

\begin{tabular}{|c|c|c|c|c|c|c|}
\hline SNP & $\begin{array}{l}\text { Genotype } \\
\text { or allele }\end{array}$ & $\begin{array}{c}\mathrm{PV}<30 \mathrm{ml} \\
\mathrm{n}=56(\%)\end{array}$ & $\begin{array}{c}\mathrm{PV} \geq 30 \mathrm{ml} \\
\mathrm{n}=53(\%)\end{array}$ & Models & OR $(95 \% \mathrm{CI})^{\mathrm{a}}$ & P-value ${ }^{a}$ \\
\hline \multirow[t]{5}{*}{ CCL2 rs 1024611} & $\mathrm{~A} / \mathrm{A}$ & $15(26.8)$ & $10(18.9)$ & Heterozygous & $1.41(0.51-3.89)$ & 0.51 \\
\hline & $\mathrm{A} / \mathrm{G}$ & $22(39.3)$ & $22(41.5)$ & Homozygous & $1.72(0.61-4.84)$ & 0.31 \\
\hline & $\mathrm{G} / \mathrm{G}$ & $19(33.9)$ & $21(39.6)$ & Dominant & $1.55(0.61-3.91)$ & 0.36 \\
\hline & A & $52(46.4)$ & $42(39.6)$ & & 1 & \\
\hline & G & $60(53.6)$ & $64(60.4)$ & & $1.36(0.79-2.36)$ & 0.27 \\
\hline \multirow[t]{5}{*}{ CCR2 rs1799864 } & $\mathrm{G} / \mathrm{G}$ & $33(58.9)$ & $30(56.6)$ & Heterozygous & $1.10(0.50-2.45)$ & 0.81 \\
\hline & $\mathrm{G} / \mathrm{A}$ & $21(37.5)$ & $21(39.6)$ & Homozygous & $1.35(0.17-10.69)$ & 0.78 \\
\hline & $\mathrm{A} / \mathrm{A}$ & $2(3.6)$ & $2(3.8)$ & Dominant & $1.12(0.52-2.44)$ & 0.77 \\
\hline & G & $87(77.7)$ & $81(76.4)$ & & 1 & \\
\hline & A & $25(22.3)$ & $25(23.6)$ & & $1.11(0.58-2.12)$ & 0.75 \\
\hline \multirow{5}{*}{ CCL5 rs2107538 } & $\mathrm{G} / \mathrm{G}$ & $34(60.7)$ & $18(34)$ & Heterozygous & $3.02(1.28-7.11)$ & 0.011 \\
\hline & $\mathrm{G} / \mathrm{A}$ & $16(28.6)$ & $27(50.9)$ & Homozygous & $2.31(0.68-7.85)$ & 0.18 \\
\hline & $\mathrm{A} / \mathrm{A}$ & $6(10.7)$ & $8(15.1)$ & Dominant & 2.83 (1.28-6.26) & 0.0094 \\
\hline & G & $84(75.0)$ & $63(59.4)$ & & 1 & \\
\hline & A & $28(25.0)$ & $43(40.6)$ & & 1.94 (1.08-3.49) & 0.027 \\
\hline
\end{tabular}

SNP, single nucleotide polymorphism; PV, prostate volume; CCL, C-C motif chemokine ligand; CCR, CC chemokine receptor; OR, odds ratio; $\mathrm{CI}$, confidence interval. ${ }^{\mathrm{a}} \mathrm{ORs}$ and P-values were calculated by logistic regression with adjustment for age. Bold text indicates a statistically significant result.

Table IX. Genotype and allele frequencies of SNPs and their association with Qmax.

\begin{tabular}{|c|c|c|c|c|c|c|}
\hline SNP & $\begin{array}{l}\text { Genotype } \\
\text { or allele }\end{array}$ & $\begin{array}{c}\mathrm{Qmax} \geq 10 \mathrm{ml} / \mathrm{sec} \\
\mathrm{n}=70(\%)\end{array}$ & $\begin{array}{c}\text { Qmax }<10 \mathrm{ml} / \mathrm{sec} \\
\mathrm{n}=39(\%)\end{array}$ & Models & OR $(95 \% \mathrm{CI})^{\mathrm{a}}$ & P-value ${ }^{a}$ \\
\hline \multirow[t]{5}{*}{ CCL2 rs 1024611} & $\mathrm{~A} / \mathrm{A}$ & $18(25.7)$ & 7 (17.9) & Heterozygous & $1.87(0.64-5.44)$ & 0.25 \\
\hline & $\mathrm{A} / \mathrm{G}$ & $25(35.7)$ & $19(48.7)$ & Homozygous & $1.25(0.42-3.79)$ & 0.69 \\
\hline & $\mathrm{G} / \mathrm{G}$ & $27(38.6)$ & $13(33.3)$ & Dominant & $1.56(0.58-4.17)$ & 0.38 \\
\hline & A & $61(43.6)$ & $33(42.4)$ & & 1 & \\
\hline & G & $79(56.4)$ & $45(57.6)$ & & $1.07(0.61-1.88)$ & 0.82 \\
\hline \multirow[t]{5}{*}{ CCR2 rs 1799864} & $\mathrm{G} / \mathrm{G}$ & $35(50)$ & $28(71.8)$ & Heterozygous & $0.38(0.16-0.92)$ & 0.031 \\
\hline & $\mathrm{G} / \mathrm{A}$ & $32(45.7)$ & $10(25.6)$ & Homozygous & $0.48(0.05-4.96)$ & 0.54 \\
\hline & $\mathrm{A} / \mathrm{A}$ & $3(4.3)$ & $1(2.6)$ & Dominant & $0.39(0.17-0.91)$ & 0.025 \\
\hline & G & $102(72.9)$ & $66(84.6)$ & & 1 & \\
\hline & A & $38(27.1)$ & $12(15.4)$ & & $0.49(0.24-1.02)$ & 0.055 \\
\hline \multirow[t]{5}{*}{ CCL5 rs2107538 } & $\mathrm{G} / \mathrm{G}$ & $34(48.6)$ & $18(46.1)$ & Heterozygous & $0.85(0.35-2.02)$ & 0.71 \\
\hline & $\mathrm{G} / \mathrm{A}$ & $29(41.4)$ & $14(35.9)$ & Homozygous & $1.74(0.52-5.83)$ & 0.37 \\
\hline & $\mathrm{A} / \mathrm{A}$ & $7(10)$ & $7(17.9)$ & Dominant & $1.02(0.46-2.27)$ & 0.96 \\
\hline & $\mathrm{G}$ & $97(69.3)$ & $50(64.1)$ & & 1 & \\
\hline & A & $43(30.7)$ & $28(35.9)$ & & $1.20(0.66-2.16)$ & 0.56 \\
\hline
\end{tabular}

SNP, single nucleotide polymorphism; Qmax, peak urinary flow rate; CCL, C-C motif chemokine ligand; CCR, CC chemokine receptor; OR, odds ratio; CI, confidence interval. ${ }^{\mathrm{O} O R s}$ and $\mathrm{P}$-values were calculated by logistic regression with adjustment for age. Bold text indicates a statistically significant result.

$\mathrm{G}>\mathrm{A}$ changes had no impact on gene expression in whole blood cells ( $\mathrm{P}=0.16$, Fig. $2 \mathrm{~A}$; and $\mathrm{P}=0.59$, Fig. $2 \mathrm{~B}$, respectively) or prostate tissues $(\mathrm{P}=0.42$ and $\mathrm{P}=0.12$, respectively; data not shown). These results suggest that the downregulation of
mRNA expression by CCL5 rs2107538 is possibly part of the molecular mechanism underlying its protective effects against BPH. Subsequently, using the RegulomeDB database, CCL5 rs2107538 was predicted to have the ability to influence the 
Table X. Genotype and allele frequencies of SNPs and their association with PSA.

\begin{tabular}{|c|c|c|c|c|c|c|}
\hline SNP & $\begin{array}{l}\text { Genotype } \\
\text { or allele }\end{array}$ & $\begin{array}{c}\mathrm{PSA}<1.5 \mathrm{ng} / \mathrm{ml}, \\
\mathrm{n}=52(\%)\end{array}$ & $\begin{aligned} \mathrm{PSA} & \geq 1.5 \mathrm{ng} / \mathrm{ml} \\
\mathrm{n} & =57(\%)\end{aligned}$ & Models & OR $(95 \% \mathrm{CI})^{\mathrm{a}}$ & P-value ${ }^{a}$ \\
\hline \multirow[t]{5}{*}{ CCL2 rs1024611 } & $\mathrm{A} / \mathrm{A}$ & $13(25)$ & $12(21.1)$ & Heterozygous & $1.01(0.37-2.75)$ & 0.99 \\
\hline & $\mathrm{A} / \mathrm{G}$ & $22(42.3)$ & $22(38.6)$ & Homozygous & $1.51(0.54-4.20)$ & 0.43 \\
\hline & $\mathrm{G} / \mathrm{G}$ & $17(32.7)$ & $23(40.4)$ & Dominant & $1.22(0.49-3.03)$ & 0.67 \\
\hline & A & $48(46.2)$ & $46(40.4)$ & & 1 & \\
\hline & G & $56(53.8)$ & $68(59.6)$ & & $1.30(0.75-2.25)$ & 0.35 \\
\hline \multirow[t]{5}{*}{ CCR2 rs 1799864} & $\mathrm{G} / \mathrm{G}$ & $32(61.5)$ & $31(54.4)$ & Heterozygous & $1.39(0.62-3.10)$ & 0.42 \\
\hline & $\mathrm{G} / \mathrm{A}$ & $18(34.6)$ & $24(42.1)$ & Homozygous & $1.24(0.16-9.78)$ & 0.84 \\
\hline & $\mathrm{A} / \mathrm{A}$ & $2(3.8)$ & $2(3.5)$ & Dominant & $1.38(0.63-3.00)$ & 0.42 \\
\hline & $\mathrm{G}$ & $82(78.8)$ & $86(75.4)$ & & 1 & \\
\hline & A & $22(21.2)$ & $28(24.6)$ & & $1.26(0.66-2.41)$ & 0.49 \\
\hline \multirow[t]{5}{*}{ CCL5 rs2107538 } & $\mathrm{G} / \mathrm{G}$ & $28(53.9)$ & $24(42.1)$ & Heterozygous & $1.50(0.65-3.45)$ & 0.34 \\
\hline & $\mathrm{G} / \mathrm{A}$ & $18(34.6)$ & $25(43.9)$ & Homozygous & $1.40(0.42-4.72)$ & 0.58 \\
\hline & $\mathrm{A} / \mathrm{A}$ & $6(11.5)$ & $8(14)$ & Dominant & $1.48(0.68-3.20)$ & 0.32 \\
\hline & $\mathrm{G}$ & $74(71.2)$ & $73(64.0)$ & & 1 & \\
\hline & $\mathrm{A}$ & $30(28.8)$ & $41(36.0)$ & & $1.30(0.72-2.32)$ & 0.38 \\
\hline
\end{tabular}

SNP, single nucleotide polymorphism; PSA, prostate-specific antigen; CCL, C-C motif chemokine ligand; CCR, CC chemokine receptor; OR, odds ratio; CI, confidence interval. ${ }^{\mathrm{a}} \mathrm{ORs}$ and P-values were calculated by logistic regression with adjustment for age.

A

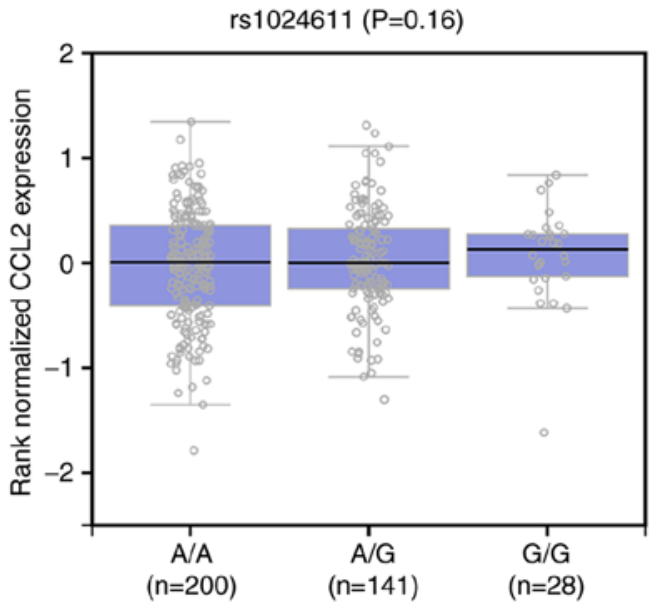

B

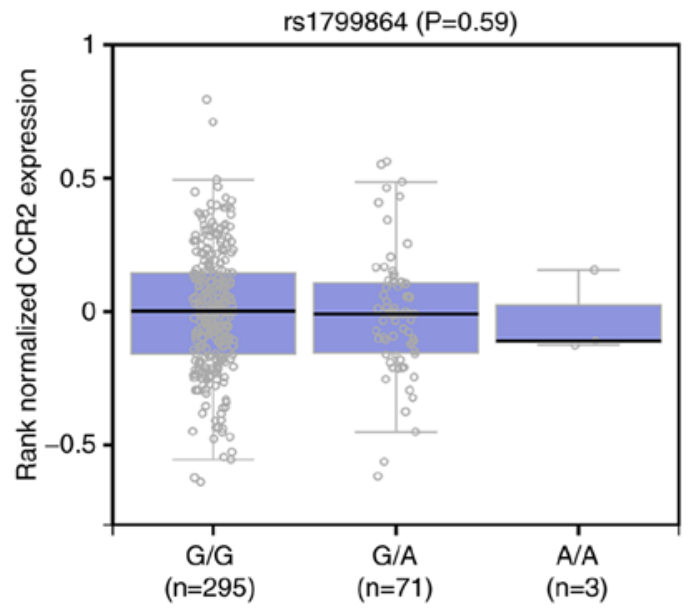

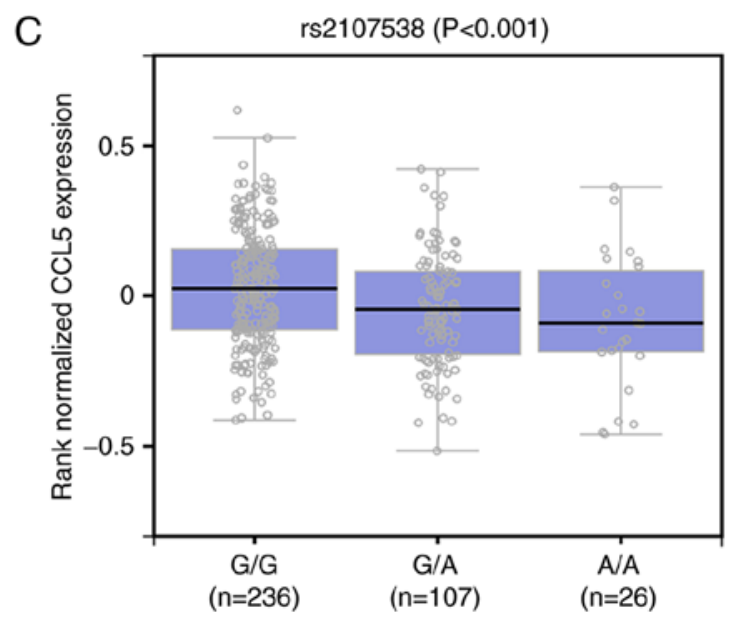

Figure 2. Roles of CCL2 rs1024611, CCR2 rs1799864 and CCL5 rs2107538 polymorphisms in the regulation of gene expression (GTEx database information for whole blood samples). (A) The CCL2 rs1024611 A>G change and (B) the CCR2 rs1799864 G>A change had no impact on gene expression. (C) The CCL5 rs2107538 G>A change downregulates the expression of CCL5 mRNA. CCL, C-C motif chemokine ligand; CCR, CC chemokine receptor. 
activity of transcription factor binding sites in its promoter region. This SNP was revealed to be on a binding site for GATA binding protein 2 (GATA2), and therefore may regulate the transcriptional activity of CCL5. This further explains the underlying mechanism for the downregulation of CCL5.

\section{Discussion}

In the present study, three common functional polymorphisms (CCL2 rs1024611, CCR2 rs1799864 and CCL5 rs2107538) were selected and their association with the risk of $\mathrm{BPH}$ development and progression was explored.

The CCL2 rs1024611 polymorphic site is located in the promoter region of the CCL2 gene, where an A>G substitution can lead to the upregulation of its expression in vivo (20). This polymorphism has been reported to participate in various diseases, such as breast cancer (7), IgA nephropathy (8) and psoriasis vulgaris (21). The association between the CCL2 rs1024611 polymorphism and prostate cancer risk has been examined in several studies, but none have been able to demonstrate a significant link $(11,13,14)$. The data analysis in the present study also demonstrated no association between CCL2 rs1024611 polymorphism and BPH susceptibility and progression, either in the genotype or the allelic form.

The CCR2 rs1799864 polymorphism is a non-synonymous mutation in exon 2, resulting in substitution of the amino acid valine with isoleucine at position 64 (22). Previous studies have reported that this genetic variation is a risk factor for psoriasis vulgaris (21), ischemic stroke (23) and carotid atherosclerosis (24). However, in the present analysis, the polymorphism rs1799864 in CCR2 was not associated with BPH susceptibility in either the genotype or the allelic form, in agreement with the study by Singh et al (25). However, it was significantly associated with IPSS and Qmax. These results suggest that the polymorphism may be associated with the severity of symptoms, rather than the development of BPH per se. In particular, the frequency of the minor allele of CCR2 rs1799864 was significantly lower in patients with high IPSS than in those with low IPSS. In addition, the G/A genotype frequency was significantly lower in $\mathrm{BPH}$ patients with low Qmax. This result suggests that patients with the A allele or G/A genotype of CCR2 rs1799864 may not manifest severe symptoms, either because the BPH is less severe or the sensitivity is less pronounced. A previous study reported that CCR2 was abundantly expressed in normal prostate tissue, and that the expression of CCR2 was elevated in the prostates of patients with BPH (26). A possible explanation of the present findings is that the CCR2 rs1799864 polymorphism may interfere with certain factors related to $\mathrm{BPH}$ progression. It may be hypothesized that CCR2 is overexpressed in BPH, but the expression level may be lower in patients with the A allele or G/A genotype of CCR2 rs1799864. Similarly, a previous study has demonstrated that individuals carrying the heterozygous (G/A) or homozygous (A/A) genotypes and the A allele of the CCR2 rs1799864 polymorphism appear to have a lower risk of cervical lesions, reflecting a protective effect against the development of cervical lesions and the susceptibility to HPV 16 infection (27).

The CCL5 rs2107538 polymorphism has been confirmed in the promoter region of the gene and is able to alter transcriptional activity and subsequent gene expression in human cell lines (28). Numerous studies have investigated the role of the CCL5 rs2107538 polymorphism in various diseases. Areeshi et al (29) performed a meta-analysis to evaluate the association between the polymorphism and tuberculosis susceptibility, and identified a significant link with increased risk of tuberculosis. In the study by Sáenz-López et al (11), the G/A + A/A genotype of CCL5 rs2107538 was reported to be significantly associated with an increased risk of prostate cancer. By contrast, a case-control study by Kidd et al (30) suggested that the inheritance of CCL5 rs2107538 (AA, $\mathrm{GA}+\mathrm{AA}$ ) was linked with a lower risk of prostate cancer. However, to the best of our knowledge, no study has been performed on the association between the CCL5 rs2107538 SNP and BPH risk. In the present study, this polymorphism was revealed to be significantly associated with BPH risk in the heterozygous, dominant and allele models. Subjects with the A allele and heterozygous G/A genotype exhibited 0.58- and 0.37-fold lower risks of developing BPH in comparison with subjects with the wild-type $\mathrm{G}$ allele and homozygous G/G genotype, respectively. In the stratification analysis, it was further observed that the frequency of the minor allele A and heterozygous G/A genotype of CCL5 rs2107538 was significantly higher in the BPH patients with a large PV than in those with a small PV. These results suggest that the CCL5 rs2107538 SNP may be a marker for protection against the occurrence of $\mathrm{BPH}$ and a risk factor for severe progression. The observed protective effects of CCL5 rs2107538 may be due to decreased transcriptional levels, decreased protein levels and, ultimately, decreased pro-inflammatory function. An analysis of the GTEx database further revealed that the mRNA expression levels were downregulated for CCL5 rs2107538 following the $\mathrm{G}>\mathrm{A}$ change in whole blood cells. Results from RegulomeDB database predicted that CCL5 rs2107538 may have the ability to influence the activity of transcription factor binding sites in its promoter region. Subsequently, we identified a binding site for GATA2, which belongs to the GATA family of nuclear regulatory proteins, and is a lineage marker serving an essential regulatory role in the development of hematopoietic (31) and genitourinary systems (32). GATA2 is overexpressed in prostate cancer (33), and is particularly upregulated in metastatic prostate cancer tissues (34). The underlying mechanism of the CCL5 rs2107538 on mRNA expression levels is possibly due to a change in the binding affinity for GATA2.

In the study by Zhernakova et al (35), this SNP was observed to correlate with a reduction in serum protein levels in patients with type 1 diabetes and controls. The findings of the present study on the protective effect of this SNP against BPH are consistent with other published reports that reveal a reduced risk of prostate cancer (30), type 1 diabetes (35) and lymphoma (36). In addition, we hypothesize that, although CCL5 may be downregulated in $\mathrm{BPH}$, the level may be higher in patients with CCL5 rs2107538 genotypes A or G/A.

Although no association of the CCL2 rs1024611 and CCR2 rs1799864 polymorphisms with BPH was observed, the joint analysis indicated that the combined occurrence of genotypes G/A-A/G of CCL5 rs2107538 and CCL2 rs1024611, and G/A-G/G of CCL5 rs2107538 and CCR2 rs1799864 were 'protective' against the occurrence of BPH. However, the G/G 
genotype of CCL2 rs1024611 may be a risk factor of BPH when combined with the G/G genotype of CCL5 rs2107538. These joint results revealed possible associations between these three genes in the risk of BPH.

The major limitation of the present study is the small sample size used for comparison. Furthermore, the functional role of CCL2 rs1024611, CCR2 rs1799864 and CCL5 rs2107538 in BPH development and progression remains poorly understood. However, to the best of our knowledge, this is the first report on the association of the CCL2 rs1024611 and CCL5 rs2107538 polymorphisms with BPH. The results revealed significant associations between the CCR2 rs1988864 and CCL5 rs2107538 polymorphisms and the development and progression of BPH. Further studies with larger sample sizes from various populations are required to elucidate the role of these polymorphisms. Knowledge of these genetic variants may be important for the development of novel treatments for BPH and improvement of the quality of life of patients with this condition.

In conclusion, the present study suggests that the CCL5 rs2107538 polymorphism may be a protective factor against the occurrence of BPH. However, subgroup analysis revealed that the CCL5 rs2107538 polymorphism may increase the risk of developing a larger PV in patients with BPH. The CCR2 rs1799864 polymorphism was revealed as a protective factor against high IPSS and low Qmax. No association was observed between the CCL2 rs1024611 polymorphism and BPH. These results suggest that CCR2 and CCL5 serve a role in BPH development and progression.

\section{Acknowledgments}

The authors would like to express their deepest gratitude to all the people who contributed to this paper.

\section{Funding}

The present study was funded by the National Natural Science Foundation of China (81672519), the Doctoral Supervisor Research Foundation of Lanzhou University Second Hospital (bdkyjj-01) and the Fundamental Research Funds for the Central Universities (lzujbky-2018-kb14).

\section{Availability of data and materials}

The datasets used and/or analyzed during the present study are available from the corresponding author on reasonable request.

\section{Authors' contributions}

YP, YG and HL were involved in conceptualization, methodology, formal analysis and investigations, writing the original draft of the manuscript and project administration. SJ, CP and WL conducted the formal analysis and investigations, data curation and project administration. YZ and HW conducted investigations and data curation. DK and RR contributed to the conceptualization, writing, reviewing and editing of the manuscript. ZW was involved in conceptualization, methodology and validation, as well as writing, reviewing and editing the manuscript, supervision and project administration. All authors read and approved the final manuscript.

\section{Ethics approval and consent to participate}

All participants provided signed informed consent for the donation of their data for clinical research. The study was conducted in accordance with the Declaration of Helsinki, and was approved by the Ethics Committee at Lanzhou University Second Hospital.

\section{Patient consent for publication}

Consent for publication was obtained from all patients.

\section{Competing interests}

The authors declare that they have no competing interests.

\section{References}

1. Thorpe A and Neal D: Benign prostatic hyperplasia. Lancet 361: 1359-1367, 2003

2. Roehrborn CG: Male lower urinary tract symptoms (LUTS) and benign prostatic hyperplasia (BPH). Med Clin North Am 95: 87-100, 2011

3. He Q, Wang Z, Liu G, Daneshgari F, MacLennan GT and Gupta S: Metabolic syndrome, inflammation and lower urinary tract symptoms: Possible translational links. Prostate Cancer Prostatic Dis 19: 7-13, 2016.

4. Fibbi B, Penna G, Morelli A, Adorini L and Maggi M: Chronic inflammation in the pathogenesis of benign prostatic hyperplasia. Int J Androl 33: 475-488, 2010.

5. Bostanci Y, Kazzazi A, Momtahen S, Laze J and Djavan B: Correlation between benign prostatic hyperplasia and inflammation. Curr Opin Urol 23: 5-10, 2013.

6. Kramer G, Mitteregger D and Marberger M: Is benign prostatic hyperplasia (BPH) an immune inflammatory disease? Eur Urol 51: 1202-1216, 2007.

7. Sambyal V, Guleria K, Kapahi R, Manjari M, Sudan M, Uppal MS and Singh NR: Association of the-2518 A/G polymorphism of MCP-1 with breast cancer in Punjab, North-West India. Asian Pac J Cancer Prev 16: 7243-7248, 2015.

8. Gao J, Liu X, Wei L, Niu D, Wei J, Wang L, Ge H, Wang M, Yu Q, Jin T, et al: Genetic variants of MCP-1 and CCR2 genes and IgA nephropathy risk. Oncotarget 7: 77950-77957, 2016.

9. Kouhpayeh HR, Taheri M, Baziboroon M, Naderi M, Bahari G and Hashemi M: CCL5 rs2107538 polymorphism increased the risk of tuberculosis in a sample of Iranian population. Prague Med Rep 117: 90-97, 2016.

10. De Nunzio C, Presicce F and Tubaro A: Inflammatory mediators in the development and progression of benign prostatic hyperplasia. Nat Rev Urol 13: 613-626, 2016.

11. Sáenz-López P, Carretero R, Cózar JM, Romero JM, Canton J, Vilchez JR, Tallada M, Garrido F and Ruiz-Cabello F: Genetic polymorphisms of RANTES, IL1-A, MCP-1 and TNF-A genes in patients with prostate cancer. BMC Cancer 8: 382, 2008.

12. Sun T, Mary LG, Oh WK, Freedman ML, Pomerantz M, Pienta KJ and Kantoff PW: Inherited variants in the chemokine CCL2 gene and prostate cancer aggressiveness in a Caucasian cohort. Clin Cancer Res 17: 1546-1552, 2011.

13. Kucukgergin C, Isman FK, Cakmakoglu B, Sanli O and Seckin S: Association of polymorphisms in $M C P-1, C C R 2$, and $C C R 5$ genes with the risk and clinicopathological characteristics of prostate cancer. DNA Cell Biol 31: 1418-1424, 2012.

14. Mandal RK, Agrawal T and Mittal RD: Genetic variants of chemokine $C C L 2$ and chemokine receptor $C C R 2$ genes and risk of prostate cancer. Tumour Biol 36: 375-381, 2015.

15. Kaplan SA, Olsson CA and Te AE: The American Urological Association symptom score in the evaluation of men with lower urinary tract symptoms: At 2 years of followup, does it work? J Urol 155: 1971-1974, 1996.

16. Homma Y, Yoshida M, Seki N, Yokoyama O, Kakizaki H, Gotoh M, Yamanishi T, Yamaguchi O, Takeda M and Nishizawa O: Symptom assessment tool for overactive bladder syndrome-overactive bladder symptom score. Urology 68: 318-323, 2006. 
17. Kaplan SA, McConnell JD, Roehrborn CG, Meehan AG Lee MW, Noble WR, Kusek JW and Nyberg LM Jr; Medical Therapy of Prostatic Symptoms (MTOPS) Research Group: Combination therapy with doxazosin and finasteride for benign prostatic hyperplasia in patients with lower urinary tract symptoms and a baseline total prostate volume of $25 \mathrm{ml}$ or greater. J Urol 175: 217-221, 2006.

18. Siami P, Roehrborn CG, Barkin J, Damiao R, Wyczolkowski M, Duggan A, Major-Walker K and Morrill BB; CombAT study group: Combination therapy with dutasteride and tamsulosin in men with moderate-to-severe benign prostatic hyperplasia and prostate enlargement: The CombAT (Combination of Avodart and Tamsulosin) trial rationale and study design. Contemp Clin Trials 28: 770-779, 2007.

19. Boyle AP, Hong EL, Hariharan M, Cheng Y, Schaub MA, Kasowski M, Karczewski KJ, Park J, Hitz BC, Weng S, et al: Annotation of functional variation in personal genomes using RegulomeDB. Genome Res 22: 1790-1797, 2012.

20. Grzegorzewska AE, Pajzderski D, Sowinska A and Jagodzinski PP: Monocyte chemoattractant protein-1 gene (MCP-1-2518 A/G) polymorphism and serological markers of hepatitis B virus infection in hemodialysis patients. Med Sci Monit 20: 1101-1116, 2014.

21. Zhang J, Song Q, Zhu K, Lu J, Xiong X and Hao F: The association of genetic variants in chemokine genes with the risk of psoriasis vulgaris in Chinese population: A case-control study. Medicine 96: e8283, 2017.

22. Nakayama EE, Tanaka Y, Nagai Y, Iwamoto A and Shioda T: A CCR2-V64I polymorphism affects stability of CCR2A isoform. AIDS 18: 729-738, 2004

23. Zhao N, Liu X, Wang Y, Liu X, Li J, Yu L, Ma L, Wang S, Zhang H, Liu L, et al: Association of inflammatory gene polymorphisms with ischemic stroke in a Chinese Han population. J Neuroinflammation 9: 162, 2012.

24. Nyquist PA, Winkler CA, McKenzie LM, Yanek LR, Becker LC and Becker DM: Single nucleotide polymorphisms in monocyte chemoattractant protein-1 and its receptor act synergistically to increase the risk of carotid atherosclerosis. Cerebrovasc Dis 28 124-130, 2009.

25. Singh V, Srivastava P, Srivastava N, Kapoor R and Mittal RD: Association of inflammatory chemokine gene $C C L 2 I / D$ with bladder cancer risk in North Indian population. Mol Biol Rep 39: 9827-9834, 2012.

26. Fujita K, Ewing CM, Getzenberg RH, Parsons JK, Isaacs WB and Pavlovich CP: Monocyte chemotactic protein-1 (MCP-1/CCL2) is associated with prostatic growth dysregulation and benign prostatic hyperplasia. Prostate 70: 473-481, 2010.
27. Santos EU, Lima GD, Oliveira Mde L, Heráclio Sde A, Silva HD, Crovella S, Maia Mde M and Souza PR: CCR2 and CCR5 genes polymorphisms in women with cervical lesions from Pernambuco, Northeast Region of Brazil: A case-control study. Mem Inst Oswaldo Cruz 111: 174-180, 2016.

28. Nickel RG, Casolaro V, Wahn U, Beyer K, Barnes KC, Plunkett BS, Freidhoff LR, Sengler C, Plitt JR, Schleimer RP, et al: Atopic dermatitis is associated with a functional mutation in the promoter of the C-C chemokine RANTES. J Immunol 164: 1612-1616, 2000.

29. Areeshi MY, Mandal RK, Panda AK and Haque S: A meta-analysis of the association between the $\mathrm{CC}$ chemokine ligand 5 (CCL5)-403 G>A gene polymorphism and tuberculosis susceptibility. PLoS One 8: e72139, 2013.

30. Kidd LR, Jones DZ, Rogers EN, Kidd NC, Beache S, Rudd JE, Ragin C, Jackson M, McFarlane-Anderson N, Tulloch-Reid M, et al: Chemokine Ligand 5 (CCL5) and chemokine receptor (CCR5) genetic variants and prostate cancer risk among men of African Descent: A case-control study. Hered Cancer Clin Pract 10: 16, 2012.

31. Zhou Y, Lim KC, Onodera K, Takahashi S, Ohta J, Minegishi N, Tsai FY, Orkin SH, Yamamoto M and Engel JD: Rescue of the embryonic lethal hematopoietic defect reveals a critical role for GATA-2 in urogenital development. EMBO J 17: 6689-6700, 1998.

32. Robinson JL, Tzou KS, Parker AS, Heckman MG, Wu KJ, Hilton TW, Pisansky TM, Schild SE, Peterson JL, Vallow LA, et al: GATA2 expression and biochemical recurrence following salvage radiation therapy for relapsing prostate cancer. Br J Radiol 90: 20170174, 2017.

33. Rodriguez-Bravo V, Carceles-Cordon M, Hoshida Y, CordonCardo C, Galsky MD and Domingo-Domenech J: The role of GATA2 in lethal prostate cancer aggressiveness. Nat Rev Urol 14: 38-48, 2017.

34. Vidal SJ, Rodriguez-Bravo V, Quinn SA, Rodriguez-Barrueco R, Lujambio A, Williams E, Sun X, de la Iglesia-Vicente J, Lee A, Readhead B, et al: A targetable GATA2-IGF2 axis confers aggressiveness in lethal prostate cancer. Cancer Cell 27: 223-239, 2015.

35. Zhernakova A, Alizadeh BZ, Eerligh P, Hanifi-Moghaddam P, Schloot NC, Diosdado B, Wijmenga C, Roep BO and Koeleman BP: Genetic variants of RANTES are associated with serum RANTES level and protection for type 1 diabetes. Genes Immun 7: 544-549, 2006.

36. Bracci PM, Skibola CF, Conde L, Halperin E, Lightfoot T, Smith A, Paynter RA, Skibola DR, Agana L, Roman E, et al: Chemokine polymorphisms and lymphoma: A pooled analysis. Leuk Lymphoma 51: 497-506, 2010. 